for prevention are more widely implemented. Our children deserve action now.

\title{
0339 BURDEN OF INJURY IN CHILDHOOD AND ADOLESCENCE IN EUROPE
}

S Polinder*, J A Haagsma, H Toet, M J P Brugmans, E F Van Beeck Correspondence: Department of Public Health, ErasmusMC, University Medical Center, Rotterdam, P.O. Box 1738, The Netherlands

10.1136/ip.2010.029215.339

Background Injury is the major cause of death and suffering among children and adolescents, but awareness of the problem and political commitment for preventive actions remain unacceptably low. We have assessed variation in the burden of injuries in childhood and adolescence in eight European countries.

Methods Hospital, emergency department and mortality databases of injury patients aged $0-24$ years were analyzed for Austria, Denmark, Ireland, Latvia, The Netherlands, Norway, Slovenia and the UK (England, Wales). Years lost due to premature mortality (YLL), years lived with disability (YLD), and disability adjusted life years (DALYs) were calculated.

Results Differences in the burden of injury in childhood and adolescence are large, with a fourfold gap between the safest countries (Netherlands and UK) in western-Europe and the relatively unsafe countries (Latvia and Slovenia) in the east. Variation between countries is attributable to high variation in premature mortality (YLL varied from 1458 per 1000 persons) and disability (YLD varied from 310 per 1000 persons). Highest burden is observed among males ages 15-24. If childhood and adolescence injuries are reduced to the level of current best injury prevention practices, 6 DALYs per 1000 child years can be avoided.

Conclusions Injuries in childhood and adolescence cause a high disability and mortality burden in Europe. In all developmental stages large inequalities between west and east are observed. Potential benefits up to almost 1 million healthy child years gained across Europe are possible, if proven ways 